EPJ Web of Conferences 47, 11002 (2013)

DOI: $10.1051 /$ epjconf/20134711002

(C) Owned by the authors, published by EDP Sciences, 2013

\title{
Transit spectroscopy with GTC
}

\author{
F. Murgas ${ }^{1, a}$, E. Pallé ${ }^{1,2}$, A. Cabrera-Lavers ${ }^{1,2}$, M.R. Zapatero Osorio ${ }^{3}$ \\ and L. Nortmann ${ }^{4}$ \\ 1 Instituto de Astrofísica de Canarias (IAC), 38205 La Laguna, Tenerife, Spain \\ ${ }^{2}$ Departamento de Astrofísica, Universidad de La Laguna (ULL), 38206 La Laguna, Tenerife, \\ Spain \\ ${ }^{3}$ Centro de Astrobiología (CSIC-INTA), 28850 Torrejón de Ardoz, Madrid, Spain \\ ${ }^{4}$ Institut für Astrophysik, Georg-August-Universität, Friedrich-Hund-Platz 1, 37077 Göttingen, \\ Germany
}

\begin{abstract}
Thanks to different ground-based surveys and space missions, nowadays we have a fairly large sample of discovered extra-solar planets to study and, without a doubt, this number will increase in the future. One of the most succesful techniques that allows us to prove the physical properties and atmospheric composition of these exoplanets is transmission spectroscopy. The level of precision that is require to measure these effects provides a technical challenge that is solved by using big telescopes and stable instruments to reach low noise levels. In this article, we will discuss the use of the $10 \mathrm{~m}$ class telescope GTC to observed planetary transits in spectroscopic mode and some of the results that we are currently obtaining.
\end{abstract}

\section{INTRODUCTION}

Currently, there are more than $850^{1}$ confirmed extra-solar planets and 2300 planetary object candidates from the Kepler mission ${ }^{2}$. Given the increasing number of exoplanetary systems found, their characterization is becoming more and more important in order to probe the vast variety of physical conditions in which they are exposed.

One of the most succesful techniques used to inferred the atmospheric composition of extra-solar planets is transmission spectroscopy. When a planet transits in front of his star, part of the starlight is absorbed by the planetary atmosphere and, since atoms and molecules absorb photons at specific energies, this results in slight differences in the observed flux at different wavelengths. As a direct consequence of these changes in flux, the depth of the transit will suffer an equivalent change in different wavelengths, helping us to deduce the presence (or absence) of several elements and molecules in the atmospheres of such exoplanets.

In order to confirm or discard the presence of elements in extra-solar planets is necessary to achieve a great accuracy in the light curve. For that reason big telescopes and stable instruments are needed. In this article, we will talk about the transmission spectroscopy technique and the current efforts that we are carrying out at the Instituto de Astrofísica de Canarias (IAC) using the 10m class telescope Gran Telescopio de Canarias.

\footnotetext{
ae-mail: murgas@iac.es

${ }^{1}$ http: //exoplanet.eu/

2 http://exoplanetarchive.ipac.caltech.edu
}

This is an Open Access article distributed under the terms of the Creative Commons Attribution License 2.0, which permits unrestricted use, distribution, and reproduction in any medium, provided the original work is properly cited. 


\section{EPJ Web of Conferences}

\section{GRAN TELESCOPIO CANARIAS}

The Gran Telescopio Canarias (GTC) is located at Observatorio Roque de Los Muchachos in La Palma, Canary Islands, Spain. Currently, GTC offers imaging and spectroscopy in mid-infrared range (CANARICAM) and imaging and spectroscopy in optical wavelengths (OSIRIS). Here, we will discuss the results obtained using the instrument OSIRIS in long-slit spectroscopic mode.

The OSIRIS instrument consists of two CCDs each with $2048 \times 4096$ pixels and a field of view (FOV) of $7.8 \times 7.8 \mathrm{arcmin}$. In its long-slit spectroscopic mode, the public available slits ranges from 0.4 to 10.0 arcsec of width, but the data that we will discuss here were taken using a custom built slit of 12 arcsec width. A wider slit allow us to reduce the possible systematic effects introduced by light losses due to imperfect guiding or changes in seeing during observations, as has been observed before using GTC OSIRIS ([2]).

For the results presented here, we used the OSIRIS R1000R grism, which has a wavelength coverage of $523-1000 \mathrm{~nm}$.

\section{METHODS}

During the observations, we place the target and a reference star in the slit so we can perform differential photometry once we extracted the spectra. The calibration and extraction of the spectrum was done using standard IRAF ${ }^{3}$ routines.

Once we obtained the time series of spectra, we proceeded to define the filters where we want to integrate the fluxes of both the target and reference star, then the light curve is created by the division of such fluxes. Figure 1a) and 1b) shows the entire range of wavelength that we can observe using GTC and an example of the integration of the flux over the full range of wavelength (white light). Under good weather conditions, we can achieve sub-mmag precisions in white light.

As we said before (see Sec. 1), to infer the presence of atoms and molecules in exoplanet atmospheres, we need to measure the depth of the transit or, equivalently, the ratio between the radius of the planet and its host $\operatorname{star}\left(R_{p} / R_{s}\right)$ with high precision at different wavelengths. With that objective in mind, we fit a transit model for each light curve and compute the level of uncertainties for the parameters fitted using a bayesian approach (see [1] for more details in the bayesian analysis).

\section{RESULTS}

\subsection{Transit color signature}

Multi-color photometry of planetary transits has some very interesting applications such as the identification of false positives in searches for exoplanets and limb darkening studies of the host star (see [3], [4] and references therein). In the example presented here (see Fig. 1c), we focus on the detection of the color signature that a transiting planet can produced in a light curve.

The color signature consists in the fact that as a planet transits the stellar disk, it occults part of the stellar light and, due to the limb darkening effect, it will block redder light in the ingress and egress than in the middle of the transit. If we are able to obtain simultaneous photometry of a transit in two bands (one blue and other red), it is possible to measure this effect using the color (Blue - Red) of the observed light curve.

Of course this transit color signature is hard to detect because, given the small comparative radius of the planet versus the stellar radius, this feature will have a small amplitude so high precision photometry and big telescopes are needed. Since the limb darkening effect is different for a planetary object than for

\footnotetext{
${ }^{3}$ IRAF is distributed by the National Optical Astronomy Observatories, which are operated by the Association of Universities for Research in Astronomy, Inc., under cooperative agreement with the National Science Foundation.
} 

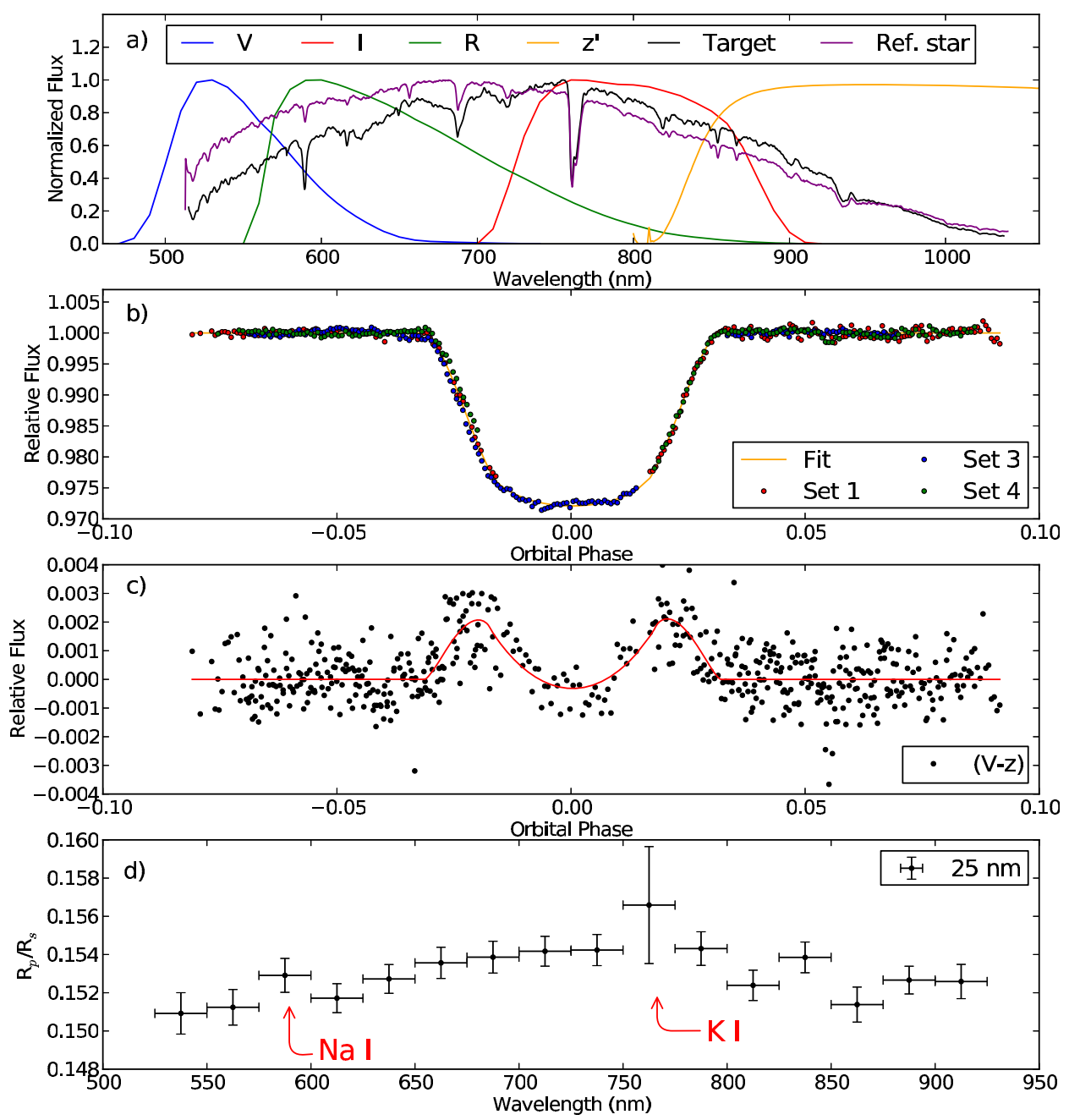

Figure 1. a) Example of a spectrum taken with GTC OSIRIS R1000R grism and showing its wavelength coverage. b) White light (full integrated spectrum) light curve for data taken in different epochs of the same transiting planet. c) Transit color signature of a planet detected using GTC; in this case we use the two bands with biggest baseline $(\mathrm{V}, \mathrm{z})$ possible; we can see that this effect is small, of about 3 mmag. d) Transmission spectrum of a transiting exoplanet showing possible detections of $\mathrm{Na}$ and $\mathrm{K}$.

a star, this effect could be used to distinguish between a transiting planet and a binary star, providing a new tool for the confirmation of planetary candidates. Another use for the transit color signature is for limb darkening studies. The comparison between the predictions of theoretical limb darkening models and observations provided by transits could help to improve current models.

In Fig. 1(c) we show an example of this color signature detected with GTC OSIRIS. The amplitude of this effect is small, of about $3 \mathrm{mmag}$ of amplitude, but nonetheless detectable with current instruments.

\subsection{Transmission spectroscopy}

In order to get the transmission spectrum of a planet we define a series of filters to integrate the spectrum of the target and reference. Once we obtain the light curve for each filter, we fit a model of the transit 
and, if that is the case, a model to take into account the systematic effects that sometimes appear in the curve. These systematics effects can be related to light losses, changing flat field with the rotation of the telescope or some other unknown effects.

In our case, we fit a model using a bayesian approach in order to obtain the probability distribution for each of the fitted parameters (depth, limb darkening, geometric parameters of the transit and systematic effects) so we can have a good estimation of the errors. The parameter that we are interested is the ratio between the planetary and stellar radius $R_{p} / R_{s}$ and its change in wavelength.

Figure 1(d) shows an example of a transmission spectrum obtained using GTC, where we integrated the observed spectrum of the host star during a transit in 10 filters of $25 \mathrm{~nm}$ width. We can see a smooth trend over the entire wavelength range except for two bins pointed out in red in the plot. These bins could be interpreted as an excess in the measured planetary radius caused by atoms of $\mathrm{Na}$ and $\mathrm{K}$, which have transitions at these wavelengths.

\section{References}

[1] F. Murgas et al., A\& A 544, A41 (2012)

[2] D. K. Sing et al., MNRAS 426, 1663S (2012)

[3] B. Tingley, A\& A 425, p.1125-1131 (2004)

[4] B. Tingley et al., A\& A 445L, 27T (2006) 